\title{
Evaluation of a concept for density measurement of solid particle flows in pneumatic conveying systems with microwaves $(8-12 \mathrm{GHz})$
}

\author{
C. Baer ${ }^{1}$, T. Musch ${ }^{1}$, M. Gerding ${ }^{1}$, and M. $\operatorname{Vogt}^{2}$ \\ ${ }^{1}$ Ruhr-Universität Bochum, Institute for Electronic Circuits, 44780 Bochum, Germany \\ ${ }^{2}$ Ruhr-Universität Bochum, High Frequency Engineering Research Group, 44780 Bochum, Germany
}

\begin{abstract}
This paper presents a novel density measuring concept for gas/coal particle compositions in pneumatic conveying systems. The proposed monitoring system uses horn antennas to perform complex electromagnetic transmittance measurements through the cross section of the conveying tube. The phase of the complex transmittance gives information about the effective permittivity, which is related to the mean volume fraction of the coal. Electromagnetic field simulations have been performed for the evaluation of the concept and the performance of the designed setup. A test stand for measurements on coal dust under reproducible conditions and with well-defined particle concentrations has been developed and implemented. The test tube has a diameter of $200 \mathrm{~mm}$ and a length of $400 \mathrm{~mm}$. Coal particles with a diameter between $20 \mu \mathrm{m}$ and $100 \mu \mathrm{m}$ have been dispersed by injecting nitrogen gas inside the test tube. Complex transmission measurements are performed in the implemented setup with a calibrated vector network analyzer within a frequency range of $8-12 \mathrm{GHz}$. Results of the conceptual evaluation by measurements with different concentrations of coal particles are presented and discussed.
\end{abstract}

\section{Introduction}

Pneumatic conveying is of interest for the handling of particulate bulk materials like grain, pellets, etc., and pulverized fuel like coal dust. Mass or volume flow rate are parameters to quantify the mass flow (Miller et al., 2000). Common methods for the measurement of the particles velocity are correlation and Doppler techniques. Besides the flow velocity, the mass density and volume concentration of the solid particles inside the conveying tube are additional parameters to be assessed. Available techniques for the monitoring

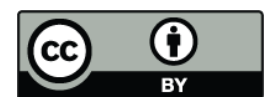

Correspondence to: C. Baer (christoph.baer@rub.de) of the volume concentration are based on cut-off frequency measurements (Conrads, 1996), determination of backscatter coefficients (Happel, 2006), different resonator properties (Penirschke and Jakoby, 2010), and various optical, acoustical, and electro-statical concepts. In this contribution, concepts for the measurement of the volume concentration by means of microwaves are discussed and evaluated. The proposed concept is based on transmission measurements through the cross-section of a conveying tube. The complex transmittance between the antennas is measured in order to determine electrical material properties of the gas/dust composition, which are representative for the mean volume fraction of the pulverized coal. The applied wide frequency range $(8-12 \mathrm{GHz})$ permits a robust and precise analysis.

\section{Monitoring sytem}

\subsection{Concept}

The most important parameter in the described application is the mass flow $\dot{m}$ of the coal particles, which is defined as:

$\dot{m}=\frac{\partial m}{\partial t}=\rho \cdot \dot{V}$

In Eq. (1), $\rho$ is the mass density of the coal. The volume flow $\dot{V}$ of the coal dust is given as follows:

$\dot{V}=\frac{\partial V}{\partial t}=\bar{v} \cdot A \cdot \bar{\zeta}$

In Eq. (2), $\bar{v}$ is the mean particle flow velocity over the crosssection of the tube, $A$ the cross-section of the tube and $\bar{\zeta}$ the mean volume fraction of the coal dust through the crosssection. By means of the subsequently described measurement concept, the mean volume fraction $\bar{\zeta}$ is measured. Figure 1 schematically shows the setup of the implemented monitoring system. Two horn antennas are arranged opposite to each other with the line-of-sight being oriented perpendicularly to the tube axis. By measuring the complex transmittance $S_{21}(\omega)$, a projection along the tube's cross-section of

Published by Copernicus Publications on behalf of the URSI Landesausschuss in der Bundesrepublik Deutschland e.V. 


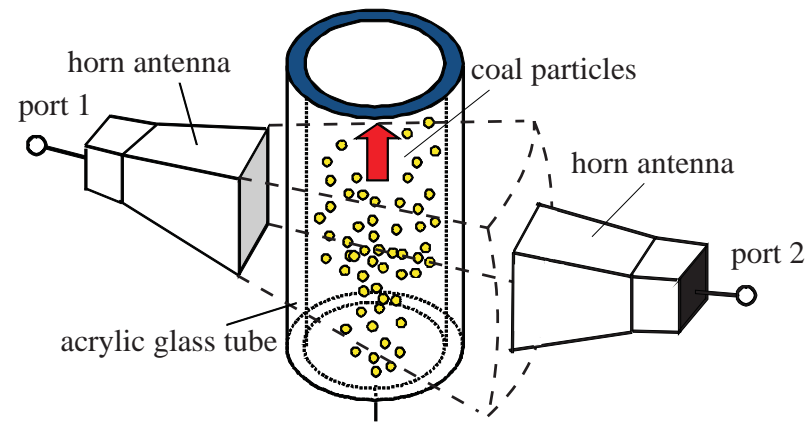

Fig. 1: Schematic of setup with conveying tube: measurement tube between two rectangular horn antennas.

the particles is obtained. With the high propagation speed of the electromagnetic waves in relation to the particle flow velocity, the particle density within the measuring tube can be assumed as static.

In order to obtain a robust model, various material properties of the coal particles have to be taken into consideration. Because the maximum diameter $K$ of the particles is less than $100 \mu \mathrm{m}$, it follows:

$K<<\lambda_{\min }$

In Eq. (3), $\lambda_{\min }$ is the smallest wavelength in the used frequency band i.e. at $12 \mathrm{GHz}$. In addition, coal can be described as a dielectric medium with a relative permittivity $\varepsilon_{\mathrm{r}, \text { coal }}$ in a range (Ruhrkohlenhandbuch, 1984):

$\varepsilon_{\mathrm{r}, \text { coal }}=2.4 \quad \ldots 2.8$

The conductivity $\sigma$ of coal is negligibly small in this application in relation to the product of angular frequency and permittivity. Consequently, the coal/gas composition can be described as a quasi-homogeneous dielectric medium described by a single effective permittivity $\varepsilon_{\mathrm{r} \text {,eff }}$ which is directly related to the mean volume fraction $\bar{\zeta}$ of the coal. Based on Sihvola (2000) the effective permittivity is given as follows:

$\varepsilon_{\mathrm{r}, \mathrm{eff}}=\varepsilon_{\mathrm{r}, \mathrm{gas}}+\frac{3 \cdot \bar{\zeta} \cdot \varepsilon_{\mathrm{r}, \mathrm{gas}} \cdot\left(\varepsilon_{\mathrm{r}, \mathrm{coal}}-\varepsilon_{\mathrm{r}, \mathrm{gas}}\right)}{\varepsilon_{\mathrm{r}, \mathrm{coal}}+2 \cdot \varepsilon_{\mathrm{r}, \mathrm{gas}}-\bar{\zeta} \cdot\left(\varepsilon_{\mathrm{r}, \text { coal }}-\varepsilon_{\mathrm{r}, \mathrm{gas}}\right)}$

By measuring the effective permittivity $\varepsilon_{\mathrm{r}, \text { eff }}$, the mean volume fraction $\bar{\zeta}$ of the coal can be obtained from Eq. (5) by taking the known permittivities into consideration. Formula (5) is known as the Maxwell-Garnett mixing formula for spherical particles (Sihvola, 2000), which is a good approximation for the description of the behavior of the coal particles.

\subsection{Measurement approach}

Under the assumption, that standing waves and multiple reflections in the system can be neglected, the phase $\phi(\omega)$ of the complex transmittance $S_{21}(\omega)$ is given as the line-integral along the transmission path between the two ports, see Fig. 1, as follows:

$$
\begin{aligned}
\phi(\omega) & =\int_{\mathrm{s}} \frac{\omega}{v_{\mathrm{ph}}(s)} d s+\phi_{0}(\omega) \\
& =\frac{\omega}{c_{0}} \cdot \int_{\mathrm{s}} \sqrt{\varepsilon_{\mathrm{r}}^{\prime}(s)} d s+\phi_{0}(\omega) \\
& =\frac{\omega}{c_{0}} \cdot \sqrt{\varepsilon_{\mathrm{r}, \mathrm{eff}}} \cdot D+\phi_{0}(\omega)
\end{aligned}
$$

By integrating the effective permittivity over the cross section a closed expression for the phase relation can be derived. In Eq. (6), $D$ is the diameter of the measuring tube, $\omega$ the angular frequency and $c_{0}$ the speed of light. The frequencydependent phase $\phi_{0}(\omega)$ denotes the wave propagation inside the antennas and feeding networks. By means of a reference measurement without any particles inside the measurement tube, the phase term $\phi_{0}(\omega)$ given in Eq. (6) can be determined:

$\phi_{\mathrm{ref}}(\omega)=\frac{\omega}{c_{0}} \sqrt{\varepsilon_{\mathrm{r}, \mathrm{gas}}} \cdot D+\phi_{0}(\omega)$

The measured phase $\phi_{\text {meas }}(\omega)$ obtained in a measurement can be eliminated for $\phi_{0}(\omega)$ as follows, by use of Eqs. (6) and (7):

$$
\begin{aligned}
\Delta \phi(\omega) & =\phi_{\text {meas }}(\omega)-\phi_{\text {ref }}(\omega) \\
& =\frac{\omega}{c_{0}} \cdot\left(\sqrt{\varepsilon_{\mathrm{r}, \mathrm{eff}}}-\sqrt{\varepsilon_{\mathrm{r}, \mathrm{gas}}}\right) \cdot D
\end{aligned}
$$

The phase $\Delta \phi(\omega)$ in Eq. (8) is a linear function of $\sqrt{\varepsilon_{\mathrm{r}, \mathrm{eff}}}$, and the mean volume fraction $\bar{\zeta}$ can be assessed by means of Eq. (5). Based on a linear regression fit of the function $\Delta \phi(\omega)$, the measurement is very robust.

\subsection{Prototype system}

A test tube for measurements on coal dust under reproducible conditions and well-defined particle concentrations has been developed and implemented. Coal particles are dispersed inside the test tube by injecting nitrogen gas.

Figure 2 shows a photo of the test stand consisting of an acrylic glass tube (I), two rectangular horn antennas (II), a nitrogen gas inlet pipe (III), and a filtering exhaust (IV). The coal particles are dispersed with a gas pressure of 5 bar. The exhausted gas is cleaned in a gas scrubbing system in order to avoid contamination. The whole test stand has a height of $80 \mathrm{~cm}$ and a diameter of $20 \mathrm{~cm}$. The complex transmittance is measured with a Rohde \& Schwarz ZVK two-port vector network analyzer.

\section{Simulations and exemplary measurements}

For the verification of the measurement concept, various simulations with a 3-D-field simulator were performed. 


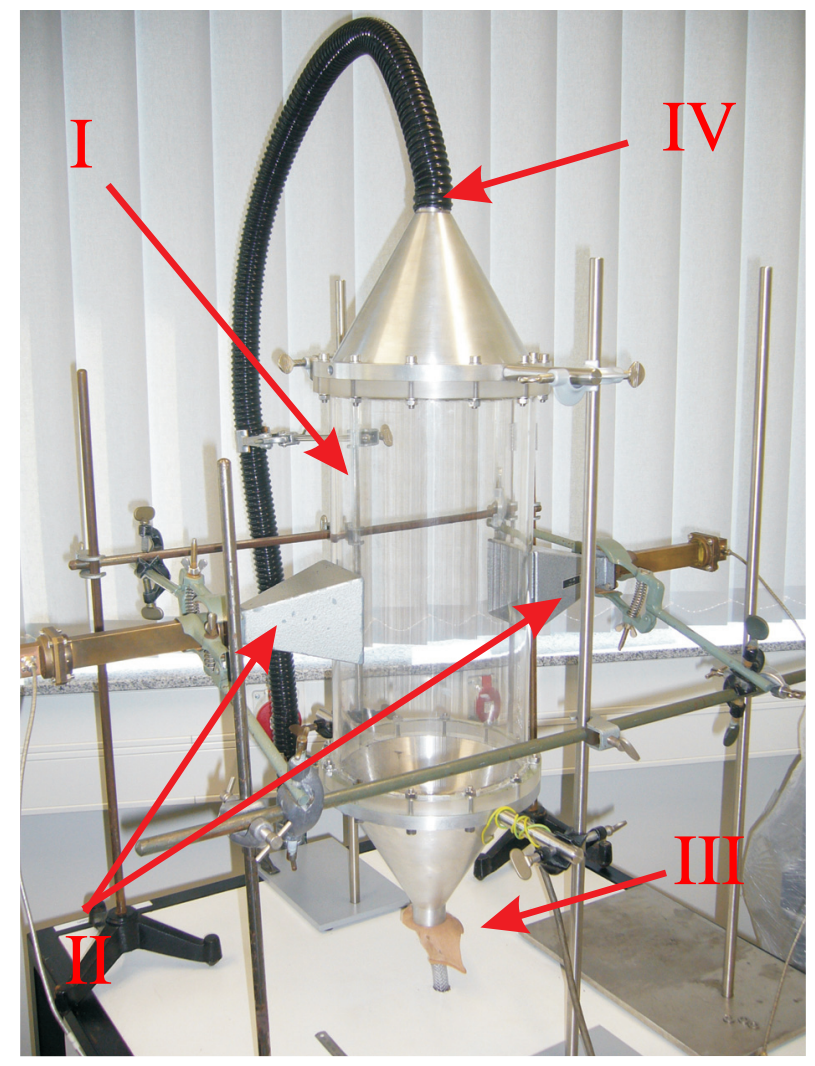

Fig. 2: Photo of the implemented test stand. I: Acrylic glass cylinder, II: Rectangular horn antennas, III: Nitrogen gas inlet pipe, IV: Filtering exhaust.

\subsection{Simulations}

Figure 3 shows simulation results for two different setups of the measuring tube. In both configurations, the measuring tube is placed in center, while the two horn antennas are arranged side wise, opposite to each other. The permittivity of the inner volume of the tube has been varied. A transient solver was used for the following simulations. While the field monitor's frequency was set to $10 \mathrm{GHz}$ in all cases, relative permittivities $\varepsilon_{\mathrm{r} \text {, eff }}=1$ and $\varepsilon_{\mathrm{r} \text {,eff }}=4$ have been used for the results in Fig. 3a and Fig. 3b, respectively. A comparison shows that the phase fronts in Fig. $3 \mathrm{~b}$ are tighter than the phase fronts in Fig. 3a. This is in agreement with the assumption that the slope of the phase line depends on the permittivity. Figures $3 \mathrm{a}$ and $3 \mathrm{~b}$ also show that multiple reflections, effected by the acrylic glass cylinder with a relative permittivity $\varepsilon_{\mathrm{r} \text {, acryl }}=3$, are negligible as they are more than $40 \mathrm{~dB}$ smaller compared to the maximum field strength.

Figure 4 shows the simulated phase $\Delta \phi$ versus frequency $f$, for increasing permittivities $\varepsilon_{\mathrm{r}}=1$ to $\varepsilon_{\mathrm{r}}=1.8$ of the inner volume. Comparing the effective permittivities from the slopes of the linear functions in Fig. 4 to the permittivities used for the simulation, an evaluation error of about $1 \%$ is obtained. This confirms the applicability of the proposed

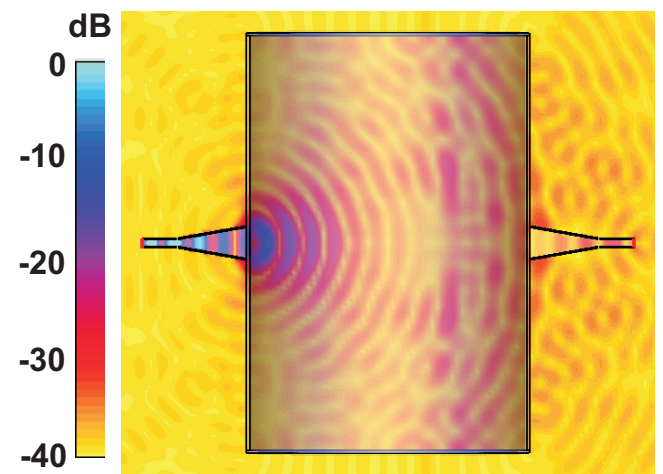

(a) 3-D simulation, magnitude of the E-field at a frequency of $10 \mathrm{GHz}$ with a homogeneous relative permittivity of $\varepsilon_{\mathrm{r}, \mathrm{eff}}=1$.

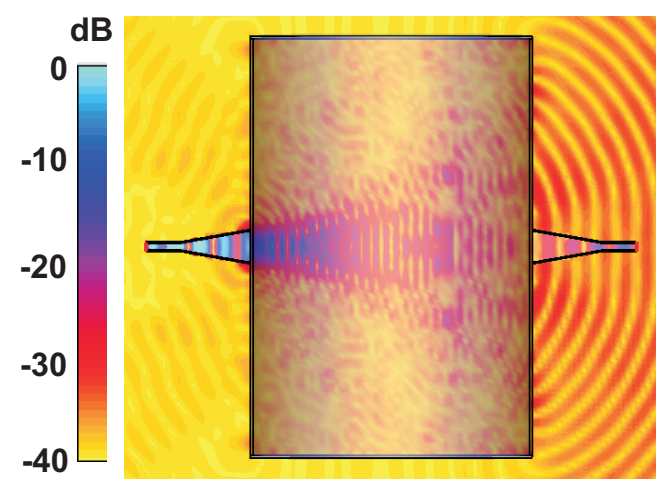

(b) 3-D simulation, magnitude of the E-field at a frequency of $10 \mathrm{GHz}$ with a homogeneous relative permittivity $\varepsilon_{\mathrm{r}, \mathrm{eff}}=4$.

Fig. 3: Screenshots of transient 3-D field simulations at constant frequency for changing permittivity, CST Microwave Studio 2010.

concept and model. Due to the linear run of the phases in Fig. 4 it can be assumed, that standing waves are also negligible as they would cause nonlinear phase runs.

\subsection{Measurements}

In a next step, measurements with different homogeneous materials with known permittivities have been performed. Two phantoms have been made of polyethylene (PE, relative permittivity $\varepsilon_{\mathrm{r}, \mathrm{eff}, \mathrm{PE}}=1.4$ ) and polyoxymethylene (POM, relative permittivity $\varepsilon_{\mathrm{r} \text {,eff,POM }}=3.8$ ). Figure 5 shows the measured phase $\Delta \phi$ versus frequency $f$ for both phantoms. Like in the simulation, the slope of the phase increases with increasing permittivity. The permittivites calculated from the slopes show a relative measurement error of $9.2 \%$ for $\mathrm{PE}$ and $0.7 \%$ for POM, respectively.

Both, simulation and phantom measurement results, confirm the validity of the proposed method. 


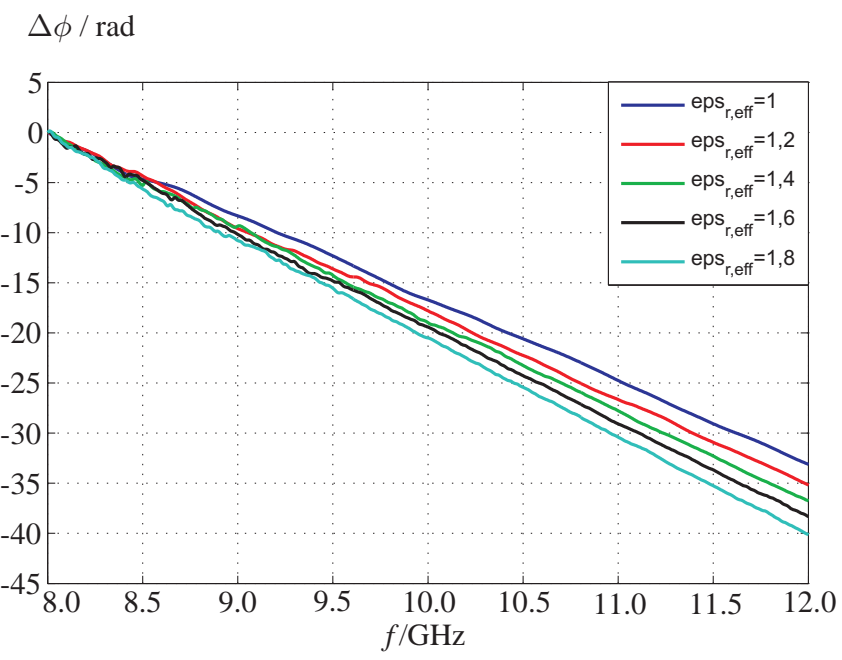

Fig. 4: Simulated phase differences $\Delta \phi$ for different homogenous permittivities.

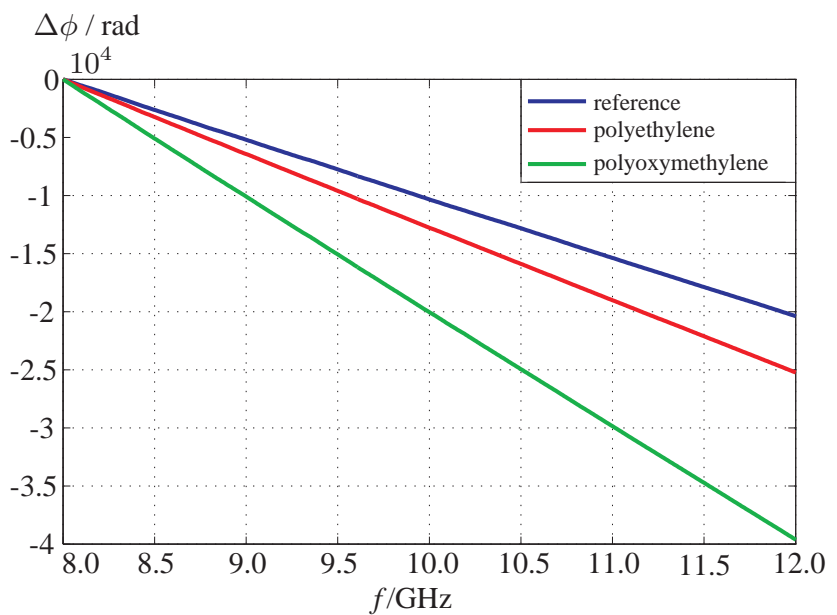

Fig. 5: Measured phase: polyethylene and polyoxymethylene phantom.

Measurements on a coal/gas composition have been performed by inserting different amounts of coal particles with well known weight into the tube. Since the total volume of the measurement tube and the density of the coal is known, measurements results can be compared to the known volume fractions. Figure 6 shows the measured total coal mass versus the actually inserted and dispersed coal mass. The relative measurement error is approximately $13 \%$.

\section{Conclusions}

In this paper, a novel density measuring method for pulverized fuels in pneumatic conveying systems is proposed. The concept is based on electromagnetic transmission measurements along the cross-section of a conveying tube consisting of acrylic glass. In the implemented setup, two measured coal /g

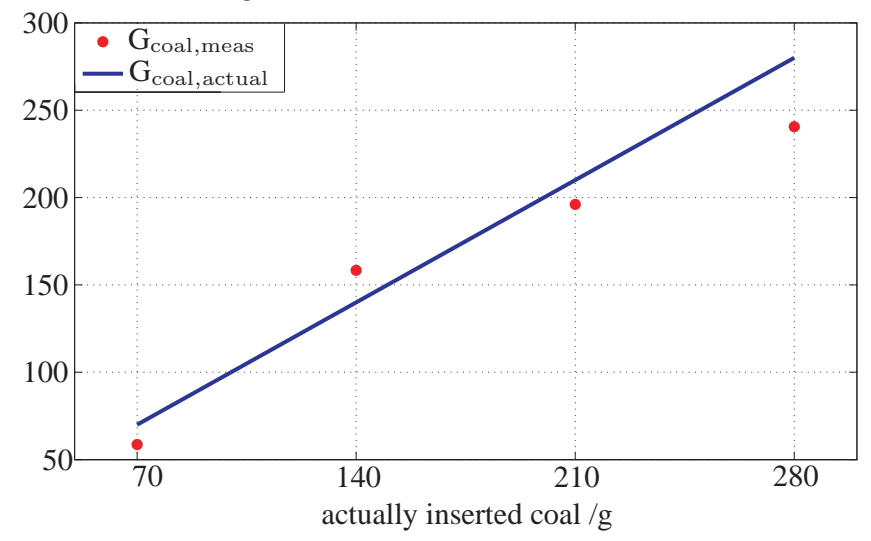

Fig. 6: Comparison between mass of coal measured inside the measurement tube and actually inserted coal mass.

horn-antennas are arranged opposite to each other with the line-of-sight being oriented perpendicularly to the tube axis. The complex transmittance between both antennas is measured in order to determine the mean volume fraction of the gas/coal particle flow. The phase of the complex transmittance contains information about the effective permittivity of the quasi-continuous medium. The phase contributions of the antennas and feeding networks are compensated by taking a reference measurement with the empty tube into account. Furthermore, electromagnetic field simulations have been performed to evaluate the concept and the performance of the method. Concerning the permittivity measurements, the evaluation error is smaller than $1 \%$. The error of measurement with two homogenous phantoms with different permittivities are $0.7 \%$ and $9.7 \%$, respectively. Different tests on dispersed coal particles revealed an error of $13 \%$. The proposed monitoring concept has been verified based on various electromagnetic simulations and measurements. The goal of future developments is to improve the measurement accuracy by employing more sophisticated calibration techniques.

\section{References}

Conrads, H. G.: Method and device for a contact-free measurement of the mass flow rate in a two phase pneumativ transport using microwaves, Eur ER Patent 07176269 A2, 1996.

Happel, J.: Method and device for measuring a mass flow, US Patent 7102133, 2006.

Miller, D., Baimbridge, P., and Eyre, D.: Technology status of PF flow measurement and control methods for utility boilers, Report No. COAL R201 DTI/PUB 00/1445, Crown Copyright 2000.

Penirschke, A. and Jakoby, R.: Design of a Moisture Independent Microwave Mass Flow Detector for Particulate Solids, GEMIC 2010.

Ruhrkohlenhandbuch: Glueckaufverlag GmbH, Essen, 1984.

Sihvola, A.: Mixing Rules with complex Dielectric Coefficients, Subsurface Sensing Technologies and Applications Vol. 1, No. 4, 2000. 